\title{
Diacronie
}

Studi di Storia Contemporanea

$\mathrm{N}^{\circ} 32,4 \mid 2017$

Proiezioni individuali e agire collettivo nella storia

\section{La rivoluzione russa e i socialisti italiani nel 1917-18}

\section{Giovanna Savant}

\section{(2) OpenEdition \\ Journals}

\section{Edizione digitale}

URL: http://journals.openedition.org/diacronie/6619

DOI: 10.4000/diacronie.6619

ISSN: 2038-0925

\section{Editore}

Association culturelle Diacronie

\section{Notizia bibliografica digitale}

Giovanna Savant, "La rivoluzione russa e i socialisti italiani nel 1917-18 », Diacronie [Online], №32, 4 | 2017, documento 5, Messo online il 29 décembre 2017, consultato il 01 mai 2019. URL : http:// journals.openedition.org/diacronie/6619; DOI : 10.4000/diacronie.6619 


\section{Diacronie}

Studi di Storia Contemporanea

\section{$32,4 / 2017$}

Proiezioni individuali e agire collettivo nella storia. Ruoli sociali, aspetti politici e nodi storiografici tra pubblico e privato

\section{La rivoluzione russa e i socialisti italiani nel 1917-18}

\section{Giovanna SAVANT}

Per citare questo articolo:

SAVANT, Giovanna, «La rivoluzione russa e i socialisti italiani nel 1917-18», Diacronie. Studi di Storia Contemporanea : Proiezioni individuali e agire collettivo nella storia. Ruoli sociali, aspetti politici e nodi storiografici tra pubblico e privato, $32,4 / 2017,29 / 12 / 2017$

URL: <http://www.studistorici.com/2017/12/29/savant_numero_32/ >

Diacronie Studi di Storia Contemporanea $\rightarrow$ http://www.diacronie.it Rivista storica online. Uscita trimestrale.

redazione.diacronie@hotmail.it

Comitato di direzione: Naor Ben-Yehoyada - João Fábio Bertonha - Christopher Denis-Delacour - Maximiliano Fuentes Codera Anders Granås Kjøstvedt - John Paul Newman - Deborah Paci - Niccolò Pianciola - Spyridon Ploumidis - Wilko Graf Von Hardenberg

Comitato di redazione: Jacopo Bassi - Luca Bufarale - Gianluca Canè - Fausto Pietrancosta - Alessandro Salvador - Matteo Tomasoni - Luca Zuccolo

Diritti: gli articoli di Diacronie. Studi di Storia Contemporanea sono pubblicati sotto licenza Creative Commons 3.0. Possono essere riprodotti e modificati a patto di indicare eventuali modifiche dei contenuti, di riconoscere la paternità dell'opera e di condividerla allo stesso modo. La citazione di estratti è comunque sempre autorizzata, nei limiti previsti dalla legge. 


\title{
5/ La rivoluzione russa e i socialisti italiani nel 1917-18
}

\author{
Giovanna SAVANT
}

Quando scoppiano i primi moti di piazza a Pietroburgo, i dirigenti del Psi hanno una conoscenza assai superficiale del socialismo russo e dei suoi contrasti interni, nonostante i contatti avuti a Zimmerwald e a Kienthal. L'imperversare della censura e la necessità di ricorrere a notizie di seconda e terza mano rendono difficile il formarsi un'idea chiara e in tempo reale di quel che accade in Russia. Contraddizioni e oscillazioni nei giudizi caratterizzano soprattutto gli articoli dell'«Avanti!», ma in realtà ogni corrente in cui è diviso il Psi tende ad accogliere e a sottolineare dei fatti russi soltanto ciò che torna utile alla propria parte nelle polemiche interne. Nel dopoguerra, la rivoluzione russa influenzerà fortemente le strategie e i programmi di ciascuna fazione.

\section{Introduzione}

Quando scoppia la Grande guerra, il PsI è tra i pochi partiti proletari a rimanere fedele al principio dell'internazionalismo e sin dall'autunno del 1914 cerca di stabilire rapporti con le minoranze socialiste degli altri paesi rimaste ostili al conflitto, allo scopo di concordare un'azione comune per la pace. Il risultato di tali sforzi è il convegno di Zimmerwald, del settembre 1915, cittadina svizzera dove si riuniscono una quarantina di delegati provenienti da tredici nazioni ${ }^{1}$. In tale occasione, gli italiani, tra cui il direttore dell'«Avanti!» Serrati e il deputato riformista Modigliani, hanno modo di conoscere personalmente Lenin e altri esponenti del socialismo russo. Fino a quel momento, il PSI non aveva che una conoscenza superficiale e frammentaria della realtà russa, della vita interna del suo movimento rivoluzionario e delle correnti che vi predominavano. A Zimmerwald, Lenin propone di trasformare la guerra imperialista in corso in guerra rivoluzionaria, ma la maggior parte dei presenti, italiani compresi, aderisce a una tesi

${ }^{1}$ Cfr. RAGIONIERI, Ernesto, «Il socialismo italiano e il movimento di Zimmerwald», in Belfagor, 2/1973, pp. 129-160. 
meno estremistica che esorta le masse a battersi per l'affermazione di principi democratici e di pacifica convivenza fra gli Stati ${ }^{2}$.

Nell'aprile del 1916, si svolge un secondo convegno a Kienthal, in cui Serrati, pur escludendo che la crisi aperta dal conflitto consenta rotture rivoluzionarie in tempi ravvicinati, si trova spesso vicino alle posizioni di Lenin ${ }^{3}$. Un mese dopo, sull'«Avanti!», due scampoli del direttore ricostruiscono brevemente la storia dei bolscevichi, paragonandoli agli intransigenti rivoluzionari italiani, mentre i menscevichi sarebbero simili ai riformisti; dagli articoli, tuttavia, emerge una certa diffidenza nei riguardi delle discussioni interne alla socialdemocrazia russa e al settarismo dei suoi leader che impedisce loro di unificarsi in un solo partito ${ }^{4}$.

Come Serrati, anche Modigliani partecipa a entrambe le riunioni, ma egli considera Lenin un utopista $^{5}$ e il suo giudizio collima con la posizione della «Critica Sociale», che, già nel luglio del 1914, aveva pubblicato un articolo sul movimento operaio russo, nettamente orientato in senso filo-menscevico, mentre i bolscevichi erano presentati come «la corrente propugnatrice del carattere cospiratorio e antioperaio del partito» ${ }^{6}$.

Nei mesi seguenti, i punti di riferimento dell'internazionalismo del PsI continuano a essere, come prima della guerra, la Francia e soprattutto la Germania, il paese dove appare più probabile l'avvento di una rivoluzione, data la presenza di un forte partito socialista e di una realtà altamente industrializzata ${ }^{7}$. La situazione interna dell'Impero russo, che peggiora progressivamente dopo l'ingresso nel conflitto mondiale, è trascurata sia dagli intransigenti sia dai riformisti, per cui lo scoppio dei moti di Pietrogrado, agli inizi del 1917, coglie di sorpresa tutti i dirigenti del partito.

\section{La rivoluzione di febbraio}

Nel Psi, l'unica personalità di spicco a commentare con entusiasmo l'avvento della rivoluzione di febbraio è, non a caso, la russa Anna Kuliscioff, in una lettera a Turati del 16 marzo 1917, giorno in cui si diffonde la notizia dell'abdicazione dello zar e della costituzione di un governo provvisorio presieduto dal principe L'vov'. Nel testo si dichiara che la rivoluzione, oltre a liberare milioni di russi dall'autarchia, potrebbe diffondersi in Germania e portare a una svolta

\footnotetext{
${ }^{2}$ Cfr. KONIG, Helmut, Lenin e il socialismo italiano. 1915/1921, Firenze, Vallecchi, 1972, p. 22.

${ }^{3}$ Cfr. RAGIONIERI, Ernesto, op. cit., p. 147.

${ }^{4}$ «La macchia si allarga» e «I Bolsceviks», in Avanti!, 16 maggio 1916.

${ }^{5}$ Cfr. ARFE', Gaetano, «Critica sociale e la Rivoluzione russa», in Critica Sociale, 10/1962, pp. 511-514.

${ }^{6}$ La C. S., «La rinascita proletaria e socialista in Russia», in Critica Sociale, 13/1914, pp. 418-421.

${ }^{7}$ Cfr. RAGIONIERI, Ernesto, op. cit., pp. 157-160.

${ }^{8}$ Sulla rivolta di Pietrogrado del febbraio 1917, si veda: GRAZIOSI, Andrea, L'Urss di Lenin e Stalin. Storia dell'Unione Sovietica 1914-1945, Bologna, Il Mulino, 2007, pp. 79-80.
} 
democratica nella storia europea ${ }^{9}$. Turati risponde seccamente che dei fatti russi «non se ne capisce niente» e avanza il sospetto che il movimento sia gestito «dai guerraioli di Russia», per continuare a oltranza la lotta contro le Potenze centrali ${ }^{10}$. La Kuliscioff replica furiosa che il neutralismo ha ormai reso «ciechi e ottusi» i socialisti italiani, poiché non comprendono che si tratta di una rivoluzione politica «in grande stile», del tutto simile a quella francese, e non di una sommossa pilotata dall'alto ${ }^{11}$. La sua ira si placa soltanto il 23 marzo, quando, alla Camera, Turati invia un saluto al popolo russo, per «il formidabile riscatto» che sta compiendo, auspicando tuttavia in modo piuttosto ambiguo che la rivoluzione possa giovare a tutti i fini, sia di guerra sia di pace ${ }^{12}$.

La cautela del leader riformista è condivisa da Serrati, che affida i primi commenti sull'«Avanti!» all'esule Vasilij Sukomlin, giunto in Italia nel $1915^{13}$. Il 17 marzo, dietro lo pseudonimo di "Junior", afferma che è iniziata la democratizzazione della Russia, ma il nuovo governo, composto soprattutto da elementi della borghesia colta, «non è espressione delle masse pietrogradesi». Le autorità militari, per conferire alla compagine ministeriale un carattere di sinistra, hanno permesso che vi entrasse in qualità di ministro della Giustizia un socialista, Kerenskij, definito come «un laburista opportunista»" ${ }^{14}$. Il giorno dopo, "Junior" riferisce soddisfatto la costituzione, come nel 1905, di un Consiglio dei delegati operai, che ottiene dal governo la promessa di convocare a breve un'Assemblea Costituente: i moti di piazza sono considerati l'inizio di un'era di lotte, che sarà contrassegnata da nuove e più radicali conquiste ${ }^{15}$. Tuttavia, le dichiarazioni del ministro degli Esteri Miljukov, appartenente al Partito dei Cadetti, d'ispirazione democratica, di voler continuare la guerra a fianco degli alleati fino alla vittoria, inducono Serrati ad affermare, il 19 marzo, che la rivoluzione russa «è stata vinta». La borghesia è riuscita «a innestare la sua azione di élite sulla folla», imbrigliando l'insurrezione, per incanalarla verso scopi non suoi: «uno sbrendolo di costituzione politica» sarà l'unico risultato dei moti popolari ${ }^{16}$.

L'editoriale serratiano fa nuovamente infuriare la Kuliscioff, che lo definisce «un articolo indecente», chiuso ai più semplici sentimenti di umanità. Trovandosi d'accordo con Treves, lo convince a preparare un pezzo per la «Critica Sociale», di plauso alla rivoluzione ${ }^{17}$. Il deputato

\footnotetext{
${ }^{9}$ Cfr. TURATI, Filippo, KULISCIOFF, Anna, Carteggio, vol. IV, 1914-1918, tomo I, Torino, Einaudi, 1977, pp. 446447.

${ }^{10}$ Ibidem, pp. 448-450.

${ }^{11}$ Ibidem, pp. 453-454.

${ }^{12}$ TURATI, Filippo, Discorsi parlamentari, vol. I., Roma, Camera dei Deputati, 1950, p. 1230.

${ }^{13}$ Cfr. CORTESI, Luigi, «Note sulle correnti del P.S.I. nel 1917 di fronte alla Rivoluzione russa», in Movimento operaio e socialista, 3-4/1968, pp. 139-199.

${ }^{14}$ JUNIOR, «Gli avvenimenti politici in Russia», in Avanti!, 17 marzo 1917.

${ }^{15} \mathrm{Cfr}$. «L'azione proletaria», in Avanti!, 18 marzo 1917.

${ }^{16}$ «Bandiera rossa!», in Avanti!, 19 marzo 1917.

${ }^{17}$ TURATI, Filippo, KULISCIOFF, Anna, op. cit., pp. 462-463.
} 
riformista dichiara che il movimento in corso non è l'espressione di una élite eroica e aristocratica, ma «una rivoluzione democratica», il cui scopo è di trasformare l'autocrazia russa in un moderno regime rappresentativo. Al termine di tale processo, il paese sarà ancora sotto la dominazione capitalistica, ma le libertà conquistate favoriranno lo sviluppo del movimento operaio. La politica interna diverrà «la palestra» che attirerà tutti gli sforzi, per cui Treves nega che l'insurrezione possa indurre il paese a intensificare il suo sforzo bellico ${ }^{18}$. La rivoluzione gioverà ugualmente all'Intesa, costituendo un esempio per i popoli degli Imperi centrali, dove «nemmeno una parcella della sua virtù di istigazione va perduta» ${ }^{19}$.

Il 9 aprile 1917, il governo russo dichiara di rinunciare a ogni espansione territoriale; tale notizia, unitamente alle dichiarazioni di Wilson per cui gli Stati Uniti combatteranno in nome di una pace equa e democratica, spingono i riformisti del PSI a ritenere che le potenze dell'Intesa non possano più essere considerate dei belligeranti allo stesso livello degli Imperi centrali: la guerra è diventata «una lotta contro un solo imperialismo, da parte di una vasta democrazia». Da questo momento, i moderati cercheranno di convincere il proletariato italiano a parteggiare apertamente per la monarchia sabauda e i suoi alleati ${ }^{20}$.

Sull'«Avanti!», il pessimismo di Serrati non è condiviso da "Junior", il quale dichiara polemicamente che la classe lavoratrice russa non permetterà alla borghesia di troncare l'opera di rinnovamento iniziata con i moti di piazza ${ }^{21}$. Egli indirizza tutte le sue simpatie verso Černov, membro del Partito socialista rivoluzionario, espressione delle grandi masse contadine e ministro dell'Agricoltura dal maggio del $1917^{22}$. "Junior" parteggia apertamente per la nuova compagine governativa e cerca di far conoscere meglio il leader russo ai lettori dell'«Avanti!», pubblicando come editoriali alcuni suoi articoli sulla necessità di nazionalizzare la terra e abolire la proprietà privata $^{23}$.

Mentre i riformisti interpretano la rivoluzione in corso come una rivoluzione democratica, il massimo organo socialista la considera una partita politica ancora aperta, suscettibile di nuovi sviluppi; tuttavia, nel PSI non manca, già in questa prima fase, chi ritiene che l'approdo degli eventi sarà senza dubbio il comunismo. In tali termini si esprime "Quidam", alias Angelo Treves, sull'«Avanti!», affermando che nell'ex Impero zarista sono i principi stessi da cui dipendono i rapporti dell'uomo con l'uomo che si stanno trasformando; le altre nazioni «non potranno restare

\footnotetext{
${ }^{18} \mathrm{NOI}$, «Primavera di rivoluzione», in Critica Sociale, 6/1917, pp. 81-83.

${ }^{19}$ TREVES Claudio, «La crisi dell'Intesa. L'Intesa e l'intervento americano», in Critica Sociale, 7-8/1917, pp. 99-101.

${ }^{20}$ Lettera di Turati a Camillo Corradini del 11 maggio 1917, in DE ROSA, Gabriele, Giolitti e il fascismo in alcune sue lettere inedite, Roma, Ed. Storia e Letteratura, 1957, p. 57.

${ }^{21}$ Cfr. JUNIOR, «Gli "elementi demagogici” e i nuovi uomini d'ordine», in Avanti!, 21 marzo 1917.

${ }^{22}$ A maggio, le dimissioni di Miljukov, che non vuole la Russia rinunci ai compensi territoriali previsti dagli accordi con gli alleati, portano alla formazione di un nuovo ministero, ancora presieduto da L'vov. Cfr. GRAZIOSI, Andrea, op. cit., pp. 85-86.

${ }^{23}$ Gli articoli di Černov sono pubblicati nei numeri del 2 e 8 aprile 1917.
} 
a lungo compresse nei vecchi istituti», quando ai loro confini tutto sarà cambiato e «in meglio» ${ }^{24}$. Sono concetti che ribadisce sulla «Critica Sociale»" ${ }^{25}$, anche se la linea ufficiale della rivista rimane quella tracciata da Claudio Treves nell'editoriale di marzo.

Tra i giovani, è Antonio Gramsci a esprimere la convinzione che il movimento proseguirà fino alla realizzazione del socialismo. Egli rifiuta il parallelo, istituito dai riformisti, con la rivoluzione francese: in Russia «non c'è il giacobinismo», termine che adopera con una forte connotazione negativa, considerandolo «un fenomeno puramente borghese». I giacobini, al regime autoritario dell'aristocrazia, sostituirono un nuovo ordine altrettanto opprimente; i socialisti russi, invece, all'autocrazia zarista hanno sostituito il suffragio universale, certi che l'ideale da essi incarnato «sia condiviso dalla maggioranza del popolo». Gramsci crede che la rivoluzione in corso rappresenti «un fatto proletario», perché sono intervenuti alcuni «fattori spirituali», che hanno prodotto un cambiamento nella morale. Per dimostrare tale asserzione, riporta la notizia di un fatto accaduto a Odessa: i rivoluzionari hanno liberato tutti i prigionieri, anche quelli colpevoli di reati comuni; ciò nonostante, alcuni decidono di restare in carcere per scontare le loro condanne fino alla fine ${ }^{26}$. Si tratta di un episodio poco rilevante, cui Gramsci conferisce una grande importanza, perché gli appare la dimostrazione di come nell'ex Impero zarista non stia soltanto cambiando il regime politico ed economico, ma vi sia un rinnovamento dei costumi e della vita morale, elementi essenziali all'interno della sua prospettiva rivoluzionaria.

Amadeo Bordiga afferma a sua volta che, nella nuova Russia, «il Terzo Stato rappresenterà ben altra parte che nella Francia dell'89» e respinge la tesi riformista che l'unico imperialismo rimasto sia quello tedesco. Al contrario, il crollo dell'autocrazia zarista dimostra che il militarismo moderno si concilia assai meglio con le più progredite democrazie, che non con gli istituti economici e politici antecedenti allo stadio capitalistico. Secondo il militante partenopeo, tre sono infatti le condizioni essenziali per il suo sviluppo: la presenza di una grande industria moderna; una organizzazione burocratica efficiente, in grado di mobilizzare l'esercito e disciplinare gli approvvigionamenti; ma soprattutto il consenso della maggioranza dei cittadini, cosa più facile da ottenere in un regime liberale aperto all'attuazione di moderate riforme sociali. Poiché tali caratteristiche mancavano del tutto alla Russia zarista, era il paese meno adatto a combattere e, infatti, «è quello che si è spezzato per primo» ${ }^{27}$.

\footnotetext{
${ }^{24}$ QUIDAM, «La luce viene dall'Oriente», in Avanti!, 6 aprile 1917.

${ }^{25}$ Si veda: TREVES, Angelo, «La Russia e il mondo», in Critica Sociale, 12/1917, pp. 158-159.

${ }^{26}$ A. G., «Note sulla Rivoluzione russa», in Il Grido del Popolo, 666, 1917; ora in GRAMSCI, Antonio, La Città futura. 1917-1918, Torino, Einaudi, 1982, pp. 138-142.

${ }^{27}$ BORDIGA, Amadeo, «Nulla da rettificare», in Avanti!, 23 maggio 1917; ora in ID., Scritti 1911-1926, vol. II. La guerra, la Rivoluzione russa e la nuova Internazionale. 1914-1918, Genova, Graphos, 1998, pp. 302-307.
} 


\section{Il governo Kerenskij e la visita degli "argonauti della pace" in Italia}

Lungo tutta l'estate del 1917, i riformisti del PsI difendono il governo russo dall'accusa di cercare la pace separata con i tedeschi. Dopo l'entusiasmo seguito alla caduta dello zar, considerato un germanofilo convinto, sulla stampa dell'Intesa è prevalsa la diffidenza verso un alleato che si rivela sempre meno propenso a combattere. Alla Camera, Treves afferma che nessun vero internazionalista può volere la pace separata, perché «è la pace di chi pensa solo per sé, agli interessi del proprio paese», mentre il resto del mondo continua «a maciullarsi» nella guerra ${ }^{28}$.

Quando, a fine luglio, si forma un nuovo ministero presieduto da Kerenskij, il deputato riformista dichiara che deve avere il pieno appoggio di tutta l'Internazionale ed esorta i compagni della frazione rivoluzionaria a non preferirgli Lenin ${ }^{29}$. Il leader bolscevico era giunto in Russia ad aprile, attraverso la Germania, e per tale ragione era considerato un agente tedesco da tutta la stampa dell'Intesa. Sull'«Avanti!», "Junior", pur difendendolo dalle accuse, lo definisce «un marxista dogmatico, eccessivamente astratto, nonché spesso intollerante e imperioso» ${ }^{30}$. A giugno, però, Sukomlin interrompe la propria collaborazione con il massimo organo socialista e, dalla Russia, arrivano alcune lettere di Angelica Balabanoff, decisamente orientate in favore dei bolscevichi. A differenza di "Junior", ella assume un atteggiamento fortemente critico verso Černov, dichiarando che il suo tentativo di conciliare il socialismo con la partecipazione al governo è condannato «a un completo insuccesso» e cancella l'illusione che il proletariato russo stesse passando di conquista in conquista attraverso la collaborazione con la borghesia ${ }^{31}$.

L'ex Impero zarista è infatti scosso da una nuova ondata di moti popolari: a giugno fallisce l'offensiva contro l'Austria e mentre l'esercito arretra di centinaia di chilometri, il governo riconosce l'indipendenza dell'Ucraina, cosa che spinge i Cadetti a dimettersi dall'esecutivo per protesta $^{32}$. Il ripetersi di nuove insurrezioni preoccupa i riformisti ${ }^{33}$, ma induce anche Serrati a ritornare al pessimismo espresso a marzo: le masse insorgono perché il governo non ha risolto nessuno dei problemi per i quali era scoppiata la rivoluzione, ovvero la guerra e la carestia. Kerenskij rappresenta «il socialismo che non ha il coraggio delle decisioni supreme ${ }^{34}$, mentre Lenin è definito «un uomo onesto, puro e forte», il quale «dettò pagine mirabili per dottrina e per

\footnotetext{
${ }^{28}$ TREVES, Claudio, Dalla Rivoluzione russa al convegno di Stoccolma, 12 luglio 1917; in ID., Come ho veduto la guerra, Roma, Rassegna Internazionale, 1921, pp. 121-140.

${ }^{29}$ Cfr. ID., «Da Parigi a Stoccolma», in Critica Sociale, 15/1917, pp. 193-195. Sulla formazione del ministero Kerenskij, si veda: CARR, Edward H., La rivoluzione bolscevica. 1917-1923, Torino, Einaudi, 1964 [Ed. Orig.: A History of Soviet Russia. The bolshevick Revolution. 1917-1923, London, MacMillan, 1950], pp. 92-94.

${ }^{30}$ JUNIOR, «Lenin», in Avanti!, 25 aprile 1917.

${ }^{31}$ BALABANOFF, Angelica, «Lettere dalla Russia», in Avanti!, 3 luglio 1917.

${ }^{32}$ Cfr. GRAZIOSI Andrea, op. cit., pp. 87-88.

${ }^{33}$ Cfr. TURATI, Filippo, KULISCIOFF, Anna, op. cit., pp. 630-631.

${ }^{34}$ «La crisi della rivoluzione», in Avanti!, 22 luglio 1917.
} 
fede $»^{35}$. In realtà, il suo pensiero e i suoi scritti sono ancora poco conosciuti in Europa, per cui le parole di Serrati esprimono semplicemente solidarietà al leader russo, simbolo di intransigenza socialista, colpito in quel periodo da un mandato di arresto da parte del governo, insieme con altri bolscevichi, per aver cercato di forzare la situazione, organizzando una manifestazione armata a Pietrogrado.

Dalla fine di luglio, Serrati si avvale dell'aiuto di un nuovo collaboratore russo, Michail Vodovosov, un emigrato socialista che si firma prima con lo pseudonimo di "Ing." e poi di "Nado" ${ }^{36}$. Nel suo primo articolo, esclude categoricamente che i bolscevichi vogliano instaurare con la forza un nuovo regime: il loro scopo è di difendere i risultati conseguiti dalle masse dai tentativi controrivoluzionari borghesi ${ }^{37}$. Nelle settimane successive, riprende a elogiare Černov, ritenendo comunque Lenin «un plus di socialismo utile in ogni caso» ${ }^{38}$.

Nel mese di agosto, giungono in Italia quattro rappresentanti del Soviet di Pietrogrado, lo stesso che ha approvato il mandato d'arresto nei riguardi dei bolscevichi. Essi cercano di ottenere l'adesione del PSI a una Conferenza internazionale da svolgersi a Stoccolma, allo scopo di raggiungere al più presto una pace generale equa e giusta ${ }^{39}$. Data la comune finalità di intenti, vengono accolti di buon grado dal governo italiano, che concede loro la possibilità di tenere conferenze pubbliche ${ }^{40}$. A Milano, sono ricevuti calorosamente dalla giunta socialista di Caldara e Turati pronuncia un discorso in cui esalta la rivoluzione nell'ex Impero zarista come quella che più di ogni altra nella storia «è vicina ai grandi ideali che i marxisti perseguono», anche se «non è ancora una rivoluzione socialista» ${ }^{41}$.

Tuttavia, nelle varie città visitate dai delegati russi, le folle li accolgono acclamando Lenin, considerato sempre più come il vero capo della rivoluzione, animato dalla volontà di andare fino in fondo, rifiutando qualsiasi soluzione di compromesso con i nemici di classe ${ }^{42}$. Il «grossolano equivoco» è denunciato dalla «Critica Sociale», che accusa la Direzione del Psi di non aver voluto spiegare alle masse che il leader bolscevico si trova in realtà all'opposizione del governo Kerenskij ${ }^{43}$. Serrati replica seccamente che Lenin è tra i pochi dirigenti socialisti a essere rimasto

\footnotetext{
${ }^{35}$ «Lenin», in Avanti!, 22 luglio 1917.

${ }^{36} \mathrm{Cfr}$. VENTURI, Antonello, Rivoluzionari russi in Italia. 1917-1921, Milano, Feltrinelli, 1979, p. 82.

${ }^{37}$ Cfr. ING., «Chiarimenti», in Avanti!, 25 luglio 1917.

${ }^{38}$ ID., «Kerenski e Lenin», in Avanti!, 31 luglio 1917.

${ }^{39}$ Cfr. CARETTI, Stefano, La Rivoluzione russa e il socialismo italiano (1917-1921), Pisa, Nistri-Lischi, 1974, pp. 7172. Si tratta di Gol'denberg, Ehrlick, Russanov e Smirnov, di varie tendenze socialiste esclusa la bolscevica. La stampa dell'Intesa li ribattezza sarcasticamente «gli argonauti della pace».

${ }^{40}$ Cfr. D'ALESSANDRO, Leonardo Pompeo, La Rivoluzione in tempo reale. Il 1917 nel socialismo italiano tra rappresentazione, mito e realtà, in DI MAGGIO, Marco (a cura di), Sfumature di rosso. La Rivoluzione russa nella politica italiana del Novecento, Torino, Accademia University Press, 2017, pp. 3-26.

${ }^{41}$ «Il discorso di Filippo Turati», in Avanti!, 13 agosto 1917.

${ }^{42}$ Cfr. CARETTI, Stefano, op. cit., pp. 74-78.

${ }^{43}$ Critica Sociale, 16/1917, p. 210.
} 
fedele ai principi della solidarietà internazionale: «le nostre folle hanno fatto del suo nome il grido di raccolta di quanti sentono oggi socialisticamente» ${ }^{44}$.

Il 13 agosto, due delegati, Gol'denberg e Smirnov, raggiungono Torino e tengono un comizio dinanzi a una folla di oltre trentamila lavoratori; Gramsci dichiara entusiasta che la rivoluzione russa è l'inizio di una nuova vita per tutti ${ }^{45}$, ma, influenzato dai giudizi dell'«Avanti!», predilige Černov: afferma infatti che Lenin è «il maestro di vita, il risvegliatore delle anime dormienti», mentre il leader del Partito socialista rivoluzionario è colui che guiderà il proletariato russo alla conquista del potere ${ }^{46}$.

Il quotidiano socialista, attraverso "Ing.", mantiene nei confronti del ministero Kerenskij un atteggiamento critico: il suo è considerato «un governo scadente, che fa una politica passivamente imperialistica e attivamente guerrafondaia $»^{47}$. Quando a settembre si verifica il tentativo di colpo di Stato del generale Kornilov, Serrati spera che si formi un nuovo esecutivo «schiettamente socialista ${ }^{48}$, ma l'entusiasmo del giornale è destinato a scemare rapidamente, perché avviene un semplice rimpasto governativo: Kerenskij conserva l'alleanza con i Cadetti ed estromette Černov, per cui il nuovo ministero appare composto «da mezzo imperialistici borghesi e mezzo socialistici imperialisti» ${ }^{49}$. Tuttavia, a ottobre, il progressivo affermarsi dei bolscevichi all'interno dei Soviet concorre a creare, tra i rivoluzionari italiani, un'atmosfera di viva attesa: Serrati abbandona ogni incertezza e afferma che Lenin «occuperà presto il posto di Kerensky» ${ }^{50}$.

In tutto il periodo che intercorre tra i moti di febbraio e l'avvento al potere dei bolscevichi, l'unico dirigente del PSI a tentare una disamina approfondita delle vicende russe è Amadeo Bordiga, direttore dell'«Avanguardia» dal settembre del 1917. Egli considera la rivoluzione come «un fenomeno di lotta tra le classi», che dura da vari decenni, e analizza le tre forze principali che agivano nell'Impero zarista: l'assolutismo, sostenuto dall'alta burocrazia e dai militari; la borghesia e il socialismo. I ceti capitalistici cercavano di spingere lo zar sulla via delle riforme, per non esasperare le masse, ma ne appoggiavano allo stesso tempo la politica estera imperialista, conforme ai propri interessi economici. Gli operai dei grandi centri urbani militavano nel Partito socialdemocratico, ma poiché il problema principale in Russia riguardava i contadini, si formò un secondo partito di ispirazione marxista, quello Socialista rivoluzionario, che puntava alla socializzazione della terra. Bordiga minimizza sulle divergenze di ordine teorico tra i due partiti,

\footnotetext{
${ }^{44}$ «Viva Lenin!», in Avanti!, 20 agosto 1917.

${ }^{45}$ Cfr. «Il compito della Rivoluzione russa», in Avanti!, 15 agosto 1917; ora in GRAMSCI, Antonio, La Città futura, cit., pp. 274-277.

${ }^{46}$ «Kerensky-Cernof», in Il Grido del Popolo, 688, 1917; ora in GRAMSCI, Antonio, La Città futura, cit., pp. 358360 .

${ }^{47}$ ING., «Cernoff e la riforma agraria», in Avanti!, 8 settembre 1917.

${ }^{48}$ «Nuovi grandi avvenimenti in Russia», in Avanti!, 11 settembre 1917.

${ }^{49}$ ING., "Socialismo e governo in Russia», in Avanti!, 16 ottobre 1917.

${ }^{50}$ [Senza titolo], in Avanti!, 13 ottobre 1917.
} 
sottolineando come fosse comune l'obiettivo di rovesciare il regime dominante con la forza. A tal fine, si proponevano di allearsi con la borghesia liberale, ma nello stesso tempo ponevano sul tappeto, accanto alla questione politica, quella economica e sociale, secondo la tattica delineata nel Manifesto ${ }^{51}$. Scoppiata la guerra, i socialisti si mantennero all'opposizione e quando, nel febbraio 1917, la carestia produsse l'insurrezione, le masse trovarono nel programma dei sovversivi «il loro naturale sbocco politico». Tutta la storia dei mesi successivi è il risultato della prova di forza tra partiti borghesi e socialisti: scrivendo l'ultimo articolo alcuni giorni dopo la presa del potere da parte dei bolscevichi, Bordiga afferma soddisfatto che il Soviet in cui sono «l'enorme maggioranza» ha finalmente assunto il potere ${ }^{52}$. L'«Avanguardia» è il giornale socialista che commenta più pacatamente di tutti la vittoria di Lenin, ritenendola «inevitabile» in base alla propria analisi condotta secondo «una rigorosa interpretazione» della dottrina marxista ${ }^{53}$.

\section{La rivoluzione d'ottobre}

Dopo la sconfitta di Kornilov, i bolscevichi aumentano il loro prestigio sui soldati e appoggiano le occupazioni di terre dei contadini poveri. Rapidamente, conquistano molti soviet urbani e a ottobre Trockij assume la presidenza di quello di Pietrogrado, formando un comitato militare rivoluzionario che può controllare oltre ventimila armati. Sebbene il consenso dei massimalisti russi non sia maggioritario nel paese, la presa del potere avviene con il sostegno silente di importanti settori della società ${ }^{54}$.

L'eco dei fatti russi appare piuttosto attutita in Italia, giungendo la notizia dell'insurrezione nello stesso periodo della rotta di Caporetto: il 9 novembre, l'«Avanti!» annuncia che i bolscevichi hanno occupato tutti i posti strategici di Pietrogrado e che Kerenskij è in fuga, ma avverte che «è impossibile determinare l'importanza e la durata degli avvenimenti attuali» ${ }^{55}$. Nei giorni successivi, la situazione appare convulsa, finché il 24 novembre, Serrati esalta la formazione di un nuovo governo rivoluzionario con a capo Lenin, mentre Trockij è commissario del popolo agli Esteri $^{56}$. In seguito, visto l'imperversare della censura, si riportano soltanto le note dell'agenzia Stefani, relative alla pubblicazione degli accordi segreti tra lo zar e gli alleati e all'avvio di trattative di pace con le Potenze centrali.

\footnotetext{
${ }^{51}$ BORDIGA, Amadeo, «La Rivoluzione russa nell'interpretazione socialista. III», in L'Avanguardia, 511, 1917; ora in ID., Scritti 1911-1926, vol. II, cit., pp. 350-352.

${ }^{52}$ ID., «La Rivoluzione russa nell'interpretazione socialista. IV» in L'Avanguardia, 512, 1917; ora in ID., op. cit. Scritti 1911-1926, vol. II, cit., pp. 352-355.

${ }^{53}$ GIPE', «Mentre Lenin trionfa ...», in L'Avanguardia, 512, 1917.

${ }^{54}$ Si veda: PONS, Silvio, La rivoluzione globale. Storia del comunismo internazionale 1917-1991, Torino, Einaudi, 2012, pp. 7-8.

${ }^{55}$ «I massimalisti padroni di Pietrogrado», in Avanti!, 9 novembre 1917.

${ }^{56} \mathrm{Cfr}$. «Lenin capo del nuovo governo», in Avanti!, 24 novembre 1917.
} 
Sulla «Critica Sociale», non appaiono commenti diretti ai fatti russi, in attesa probabilmente che le cose si chiariscano; tuttavia, nell'incertezza dei primi momenti successivi alla vittoria bolscevica, Turati arriva a ipotizzare che il colpo di Stato sia stato concertato tra Kerenskij e Lenin: poiché il paese non è più in grado di combattere, il capo del governo ha lasciato il potere nelle mani dei massimalisti, affinché siano loro a stipulare la pace separata, «salvo riprendere il timone più tardi» ${ }^{57}$.

A metà dicembre, l'«Avanti!» pubblica due lunghi editoriali per cercare di interpretare le vicende russe, iniziando così un dibattito sulla possibilità di instaurare il comunismo in un paese economicamente arretrato come l'ex Impero zarista. Il primo intervento è di Carlo Bertani, secondo il quale la rivoluzione risente, oltre che degli influssi di Marx, anche di quelli di Tolstoj e di Bakunin. Egli sostiene che la socializzazione delle industrie potrà essere realizzata rapidamente, in quanto la guerra ha favorito la concentrazione dei capitali nelle mani di pochi, che saranno esautorati facilmente; maggiori problemi presenterà invece la nazionalizzazione delle terre, perché gran parte dei contadini aspirano a diventare piccoli proprietari ${ }^{58}$.

Il secondo editoriale è di Gramsci, provocatorio sin dal titolo, perché definisce la rivoluzione russa come «la rivoluzione contro il Capitale» di Marx: tale libro, nell'Impero zarista, era letto soprattutto dai borghesi, perché era la dimostrazione «della fatale necessità» che anche qui si formasse una civiltà capitalistica, prima che la classe lavoratrice cominciasse a pensare alla propria riscossa. In realtà, massimo fattore di storia è l'uomo o meglio «le società degli uomini», che sviluppano una volontà collettiva, la quale diventa a sua volta «la plasmatrice della realtà oggettiva». In condizioni di pace, tale volontà si forma lentamente, ma quando il ritmo dei fatti è alterato da un evento imprevedibile come la guerra, allora la lotta di classe può subire una brusca svolta: le sofferenze e i rischi condivisi quotidianamente, per tre anni, nelle trincee e nelle caserme, hanno mutato la psicologia delle masse, favorendo la nascita di sentimenti solidaristici sui quali si è innestata la propaganda bolscevica, permettendo così il salto dall'economia patriarcale a quella socialista ${ }^{59}$.

L'editoriale gramsciano suscita aspre polemiche sia tra i riformisti, sia nella corrente rivoluzionaria. Treves pubblica sulla «Critica Sociale» una lettera del leader dei menscevichi Martov - avversario di Lenin e del suo tentativo di introdurre il socialismo in Russia - affermando di condividerne la posizione. Il dirigente riformista accusa Gramsci di fuorviare la dottrina di

\footnotetext{
${ }^{57}$ TURATI, Filippo, KULISCIOFF, Anna, op. cit., pp. 746-749.

${ }^{58}$ Cfr. BERTANI, Carlo, «L'esperimento socialista russo», in Avanti!, 17 dicembre 1917.

${ }^{59}$ A. G., «La rivoluzione contro il Capitale», in Avanti!, 24 dicembre 1917; ora in GRAMSCI, Antonio, La Città futura cit., pp. 513-517.
} 
Marx, ritenendo che sia sufficiente la volontà degli individui per far compiere balzi alla storia, mentre nessun decreto di Lenin «può sorvolare i periodi dell'evoluzione della proprietà» ${ }^{60}$.

Gramsci, a sua volta, ritiene che i riformisti abbiano ridotto il marxismo a «dottrina dell'inerzia del proletariato» ${ }^{61}$. Egli chiarisce tuttavia che i bolscevichi non mirano a realizzare subito una società in cui tutti gli uomini siano divisi in due classi, perché tale obiettivo si può raggiungere solo quando lo sviluppo economico abbia raggiunto il suo culmine. Istituire il socialismo però significa «anche altro» $\mathrm{e}$, nel caso russo, vuol dire abolire i vecchi istituti giuridici e i privilegi a essi connessi, chiamando «all'esercizio della sovranità statale tutti gli uomini». I cambiamenti politici influiscono a loro volta sull'economia, in modo che la produzione si svolge con «fini socialisti», anche se per molto tempo ancora non potrà offrire quel benessere che in regime collettivista tutti gli uomini dovrebbero avere ${ }^{62}$.

La lettera di Martov è pubblicata anche dall'«Avanti!», con una postilla di Serrati, il quale asserisce di condividere pienamente la linea politica di Lenin e minimizza sulle accuse di terrorismo avanzate contro i bolscevichi, ritenendole espressione del «gusto innato» dei socialisti russi di litigare tra loro ${ }^{63}$. Anzi, il giornale non esclude che, trascorso il periodo della dittatura del Commissariato del Popolo, la nuova Russia si dia «un governo di concentrazione socialista da Lenin, attraverso Martov, a Cernoff», per procedere alla soluzione dei grandi problemi di riorganizzazione interna ${ }^{64}$. Inoltre, osserva come il leninismo, più che una dottrina, «sia soltanto un metodo, anzi appena una forma di lotta del proletariato internazionale», affermatosi concretamente in Russia ${ }^{65}$. Serrati ne apprezza l'opposizione alla guerra e l'intransigenza verso ogni governo borghese, ma non ne coglie la complessità ideologica, per cui il maggior contributo all'analisi teorica della rivoluzione bolscevica proviene dalle ali estreme del partito: i riformisti e gli esponenti della frazione intransigente di Bordiga.

Tra i massimalisti, è proprio il militante partenopeo a prendere le distanze dalle affermazioni gramsciane, ritenendo che non possa essere considerato «antimarxistico l'inizio della rivoluzione socialista nel paese ove non si è ancora compiuta quella borghese». Egli crede che la Russia del 1917 sia simile alla Germania del 1847: un paese quasi feudale, in attesa di un vero regime borghese, ma nel quale Marx ed Engels ritenevano possibile l'avvento del comunismo ${ }^{66}$. L'azione di Lenin gli appare conforme ai dettami del Manifesto e, anche in seguito, Bordiga tenderà a credere che le vicende russe si sviluppino lungo una linea chiara e semplice, di cui è possibile

\footnotetext{
${ }^{60}$ VERY-WELL, «Lenin, Martoff e ... noi!», in Critica Sociale, 1/1918, pp. 4-5.

${ }^{61}$ A. G., «La Critica critica», in Il Grido del Popolo, 703, 1918; ora in ID., La Città futura cit., pp. 554-558.

${ }^{62}$ «L'ultimo tradimento», in Avanti!, 3 gennaio 1918; ora in Ibidem, pp. 536-538.

${ }^{63}$ «La lotta delle tendenze nel socialismo russo», in Avanti!, 25 dicembre 1917.

${ }^{64}$ I. O., «I casi dell'Ucraina», in Avanti!, 28 dicembre 1917.

${ }^{65}$ «La storia del Prof. Ciccotti», in Avanti!, 19 gennaio 1918.

${ }^{66}$ BORDIGA, Amadeo, "Gli insegnamenti della nuova storia» in Avanti!, 16 febbraio 1918; ora in ID., Scritti 1911-1926, vol. II, cit., pp. 411-422.
} 
prevedere ogni punto di arrivo, svilendo così il significato di fatto nuovo assunto dalla rivoluzione bolscevica.

La distanza interpretativa sugli eventi russi, tra riformisti e rivoluzionari, è ormai netta: l'avvento al potere di Lenin e dei suoi compagni divide le due anime del PsI in modo irrimediabile; da questo momento, i massimalisti guarderanno alla Russia dei Soviet come a un modello da imitare, mentre per i moderati essa non costituisce che un'aberrazione dei canoni del materialismo storico.

\section{Lo scioglimento della Costituente e il trattato di pace di Brest-Litovsk}

Nel gennaio del 1918, i bolscevichi decidono di sciogliere la neoeletta Assemblea Costituente in cui risultano essere in minoranza rispetto ai socialisti rivoluzionari ${ }^{67}$. L'«Avanti!» si limita a riportare la notizia senza commenti, ma, pungolato dai nazionalisti, Serrati dichiara di non aver preso troppo sul serio l'informazione della Stefani, «agenzia di notizie adulterate», e riporta una serie di errori riguardanti la Russia divulgati negli ultimi mesi ${ }^{6}$. In precedenza, il massimo organo socialista aveva considerato favorevolmente la convocazione di un'Assemblea Costituente da eleggere con suffragio universale, ritenendola «la futura vera rappresentanza del popolo russo» ${ }^{69}$. Probabilmente, il suo scioglimento lascia inizialmente perplesso lo stesso Serrati ed è Gustavo Sacerdote, corrispondente dalla Svizzera, ad assumersi il compito di difendere i bolscevichi. Egli afferma che i massimalisti russi sono una minoranza mentre, secondo Marx, la dittatura del proletariato dovrebbe essere la dittatura della maggioranza sfruttata, ma si domanda, assai spregiudicatamente: «occorre sempre giurare sulle parole del Maestro?». Inoltre, non esclude che, com'è accaduto altre volte nella storia, «le minoranze di oggi diventino le maggioranze di domani» ${ }^{70}$.

A difendere con forza l'operato dei bolscevichi provvedono nuovamente Gramsci e Bordiga. Il polemista sardo osserva che la Costituente era una forma di parlamento tipica delle democrazie borghesi e non adatta in quanto tale alla costruzione di un nuovo ordine sociale. Lenin e i suoi compagni esercitano «provvisoriamente la dittatura» per permettere alla maggioranza di instaurare il proprio regime "all'infuori di ogni apriorismo» ${ }^{71}$. Indubbiamente, Gramsci tende a

\footnotetext{
${ }^{67}$ Cfr. GRAZIOSI, Andrea, op. cit., p. 96.

${ }^{68}$ «Polemichette», in Avanti!, 27 gennaio 1918.

${ }^{69}$ ING., «L'Assemblea Costituente», in Avanti!, 5 luglio 1917. Ancora a dicembre, "Ing." riteneva infondate le voci secondo cui i bolscevichi avrebbero voluto sciogliere l'Assemblea, dove erano in minoranza. Cfr. ING., «La Costituente», in Avanti!, 19 dicembre 1917. Dalla fine di gennaio, "Ing." dirada la sua collaborazione con l'«Avanti!», perché, in conflitto con Serrati, continua a prediligere Černov. Cfr. CORTESI, Luigi, Note sulle correnti del PSI, cit., p. 145.

${ }^{70}$ GENOSSE, «Il programma dei bolscevichi», in Avanti!, 8 febbraio 1918.

${ }^{71}$ «Costituente e Soviety», in Il Grido del Popolo, 705, 1918; ora in GRAMSCI, Antonio, La Città futura, cit., pp.
} 
formarsi una immagine piuttosto idealizzata del processo in corso in Russia: dichiara, infatti, che i bolscevichi concepiscono l'azione politica come «un processo infinito di perfezione», perché la loro cultura «è materiata di filosofia storicistica». Tale indirizzo mentale si diffonde tra i proletari, i quali diventano così sempre più capaci di controllare l'operato degli organi esecutivi, permettendo di fatto una compartecipazione dei governati al potere ${ }^{72}$.

Anche Bordiga considera la Costituente un tipo di parlamento borghese, ma mentre Gramsci insiste sul carattere transitorio della dittatura, Bordiga dichiara che la rivoluzione si sta svolgendo in due tempi, rigidamente distinti: dopo la conquista violenta del potere, avvenuta rapidamente, è iniziata la trasformazione degli istituti sociali, la quale richiederà un lungo periodo di coercizione, necessario per sopprimere con la violenza tutti gli ostacoli, per cui la dittatura bolscevica potrà durare anche diverse generazioni. Poiché il processo che conduce all'instaurazione del comunismo è autoritario, Bordiga ritiene che Marx ed Engels errassero, attribuendo una importanza eccessiva al suffragio universale e ad altri meccanismi democratici ai fini del successo della rivoluzione ${ }^{73}$.

Per quanto riguarda i riformisti del PSI, non si trovano nell'immediato commenti di aperta condanna dello scioglimento della Costituente: come spiegherà più tardi Turati, le notizie frammentarie e non di rado distorte che arrivavano dalla Russia «dovettero lasciarci molto scettici e non credemmo di doverle raccogliere e commentare», convinti che gli inizi di ogni rivoluzione raramente si disgiungono da violenze e repressioni sanguinose $\mathrm{e}^{74}$.

Tutta la stampa socialista segue invece con attenzione l'andamento delle trattative per un armistizio tra la Russia e le Potenze centrali. Secondo Bordiga, i bolscevichi, ordinando la smobilitazione dell'esercito, hanno distrutto il militarismo zarista, ma hanno anche svuotato di contenuto quello tedesco: gli Imperi centrali non possono più servirsi dello «spauracchio russo» per spingere all'azione violenta il popolo ${ }^{75}$. Egli è certo che i massimalisti non firmeranno la pace, finché non saranno liberati i territori invasi, e che i soldati tedeschi non ubbidiranno all'ordine di avanzare, ma pecca di eccessivo ottimismo: infatti, subito dopo la rottura delle trattative, gli Imperi centrali riprendono le operazioni militari, procedendo senza incontrare alcuna resistenza. Il governo russo è costretto a ripristinare i contatti diplomatici, che si concludono il 3 marzo 1918, con il trattato di Brest-Litovsk: il nuovo regime dei Soviet deve rinunciare a vaste porzioni del proprio territorio, oltre a dover corrispondere un'altissima indennità di guerra ${ }^{76}$.

602-603.

${ }^{72}$ Cfr. «Per conoscere la Rivoluzione russa», in Il Grido del Popolo, 726, 1918; ora in GRAMSCI, Antonio, Il nostro Marx. 1918-1919, Torino, Einaudi, 1984, pp. 131-139.

${ }^{73}$ BORDIGA, Amadeo, «Gli insegnamenti della nuova storia», in Avanti!, 16 febbraio 1918; ora in ID., op. cit., pp. 411-422.

${ }^{74}$ TURATI, Filippo, «Il terrore», in Critica Sociale, 19/1918, pp. 222-223.

${ }^{75}$ BORDIGA, Amadeo, «Gli insegnamenti della nuova storia», cit.

${ }^{76}$ Sul trattato di pace di Brest Litovsk, si veda: GRAZIOSI Andrea, op. cit., p. 104. 
Sull'«Avanti!», Serrati denuncia il carattere imperialista della pace: i bolscevichi hanno dovuto rinunciare a uno dei principi cardine di Zimmerwald, quello dell'autodecisione dei popoli, fatto assai grave, perché «non è possibile transigere» con uno solo di tali postulati senza «svisare lo spirito» della ricostruzione sociale tratteggiata durante il convegno svizzero. In ogni caso, egli è certo che le conquiste della forza bruta non saranno durevoli, perché le masse imporranno una revisione di tutti i trattati, dopo le conferenze della diplomazia ufficiale ${ }^{77}$.

I riformisti considerano la resa come una pausa necessaria per riorganizzare le forze in vista di una ripresa della guerra contro gli invasori: in tal senso si esprime Modigliani alla Camera, paragonando i bolscevichi ai comunardi francesi, in quanto la loro azione coniuga il proposito rivoluzionario con l'obiettivo di salvaguardare la vita futura del proprio paese. Per i socialisti, l'indipendenza e l'integrità della nazione «sono una premessa storica necessaria della lotta sociale del proletariato». In tal modo, Modigliani stabilisce una analogia diretta tra Brest Litovsk e Caporetto, cercando di propagandare il principio che sia dovere dei socialisti difendere il territorio nazionale ${ }^{78}$.

Bordiga nega recisamente tale interpretazione dei fatti e osserva che le speranze dei bolscevichi sono naufragate, perché gli Imperi centrali hanno ottenuto l'alleanza della Finlandia e dell'Ucraina contro la Russia. Lenin e i suoi compagni hanno quindi accettato le dure condizioni di pace, in attesa della «conversione» del popolo tedesco che cancellerà i trattati imperiali. Dinanzi alle obiezioni avanzate sia dai riformisti sia da Serrati che le condizioni di pace violano il diritto dei popoli a disporre di se stessi, sancito a Zimmerwald, Bordiga dichiara che in realtà quella formula non rappresenta «che un lato secondario (e superfluo) del pensiero degli internazionalisti adunati»: essi vollero indicare una soluzione del conflitto meno difforme dagli interessi del proletariato mondiale, nell'ipotesi che la conflagrazione si concludesse permanendo ancora il regime capitalistico, ipotesi da cui la Russia è uscita ${ }^{79}$.

Con la sua disamina, il militante partenopeo intende abbattere il dogma riformista della guerra di difesa nazionale, ma non coglie il carattere compromissorio dell'accordo e lo ascrive a una decisione ideologica dei bolscevichi: a uno Stato proletario non è lecito, per principio, entrare in conflitto con uno Stato capitalistico, pena il suo suicidio politico. In realtà, Lenin voleva evitare che i tedeschi provocassero la fine della rivoluzione, infliggendo ai russi nuove sconfitte. Tale realismo del leader bolscevico è colto con precisione da Gramsci, il quale comprende che il problema dei rapporti russo-tedeschi era essenzialmente un problema di rapporti tra due forze: quella degli Imperi centrali, «in piena efficienza combattiva e aggressiva» e quella del regime dei

\footnotetext{
${ }^{77}$ «E dopo la pace di Brenno?», in Avanti!, 5 marzo 1918.

${ }^{78}$ MODIGLIANI, Giuseppe Emanuele, Discorsi Parlamentari, Roma, Camera dei Deputati, 1955, pp. 500-506.

${ }^{79}$ A. B., «Le direttive della Rivoluzione russa in una fase decisiva», in Avanti!, 24 maggio 1918; ora in BORDIGA, Amadeo, Scritti 1911-1926, vol. II, cit., pp. 426-435.
} 
Soviet, «in dissoluzione». In una situazione simile, non si poteva che accettare la pace, a qualunque condizione fosse imposta ${ }^{80}$.

\section{Contro "la crociata antibolscevica"}

Lo scioglimento dell'Assemblea Costituente, la pace di Brest-Litovsk e le requisizioni violente del grano nelle campagne, necessarie per far fronte alla crisi alimentare delle città, contribuiscono a far precipitare la Russia nella guerra civile ${ }^{81}$. I bolscevichi cercano di schiacciare ogni forma di opposizione e non meraviglia che i riformisti del PsI li attacchino sempre più duramente, accusandoli di seguire Bakunin piuttosto che Marx. Treves afferma che Lenin e Trockij hanno instaurato una dittatura personale, creando "un bonapartismo anarchico» incompatibile con la vera essenza del socialismo ${ }^{82}$. Le simpatie della «Critica Sociale» sono tutte per i menscevichi e per altri marxisti moderati come Plechanov, tra i fondatori, nel 1898, del Partito socialdemocratico russo. Quando nel giugno del 1918 giunge la notizia della sua morte, Turati lo elogia come colui che combatté contro le forme di socialismo mistico diffuse nell'Impero zarista. Dopo l'avvento al potere di Lenin e dei suoi compagni, però, Plechanov venne «scavalcato» dagli eventi, o per meglio dire «furono i fatti a deviare dalla linea logica che avrebbero dovuto percorrere». Secondo Turati, i bolscevichi rappresentano un ritorno a quelle forme di socialismo utopistico che Plechanov ha sempre osteggiato e avanza il sospetto che la sua morte sia stata provocata dal «contrasto violento fra le sue speranze e il destino» ${ }^{83}$.

Nel ribattere sull'«Avanti!», Gramsci afferma che la guerra è stata l'evento «che ha sostanziato di necessità la dittatura proletaria», guerra che la Russia zarista ha dovuto combattere nelle stesse forme degli Stati capitalistici più progrediti. Il conflitto in corso, oltre a richiedere una industria moderna, ha bisogno di uomini che siano dotati della resistenza spirituale necessaria per combattere e sostenere sacrifici durissimi. Tale umanità si trova più facilmente in Germania e in Inghilterra, le due potenze maggiormente industrializzate che si fronteggiano nelle trincee: la massa dei soldati, da entrambi le parti, è composta di operai, «rinsaldati cerebralmente e fisicamente dall'officina e dalla macchina». La Russia, invece, è crollata, perché con un esercito formato in gran parte da contadini e con un'economia arretrata, non poteva combattere una guerra di tecnica e di organizzazione come gli altri Stati più evoluti. Nel caos generale, i pochi

\footnotetext{
${ }^{80}$ «Per conoscere la Rivoluzione russa», in Il Grido del Popolo, 726, 1918; ora in GRAMSCI, Antonio, Il nostro Marx, cit., pp. 131-139.

${ }^{81}$ Si veda: PONS, Silvio, op. cit., p. 14.

${ }^{82}$ VERY-WELL, «Menscevichi contro bolscevichi», in Critica Sociale, 7/1918, pp. 78-80.

${ }^{83}$ TURATI, Filippo, «Giorgio Plekhanow», in Critica Sociale, 12/1918, p. 143.
} 
borghesi presenti cercarono di imporsi, ma «furono travolti» e il proletariato assunse la direzione della vita politica ed economica del paese ${ }^{84}$.

Il polemista sardo ammette tuttavia che un nuovo ordine stenta ad affermarsi in Russia, ma ciò è naturale: «non si crea una società umana in sei mesi, quando tre anni di guerra hanno esaurito un paese, l'hanno privato dei mezzi meccanici per la vita civile». I riformisti chiedono ai bolscevichi quello che non si è mai domandato agli altri rivoluzionari del passato, ovvero «la creazione fulminea», immediata di una nuova civiltà ${ }^{85}$.

I moderati rimangono comunque contrari all'esperimento russo e dalla seconda metà del 1918 e fino all'avvento del fascismo, la «Critica Sociale» pubblica con assiduità articoli di socialisti avversari dei bolscevichi, tra cui l'ex collaboratore dell'«Avanti!» "Junior", affinché i lettori italiani sappiano degli sforzi che essi fanno per salvare il paese «dal dispotismo leniniano» ${ }^{86}$.

La maggioranza del PSI, invece, esalta gli aspetti più classisti e internazionalisti della rivoluzione bolscevica, in modo da avere maggiori argomenti nella polemica interna contro le tendenze collaborazioniste dei riformisti, divenute sempre più marcate dopo la rotta di Caporetto. Tuttavia, l'«Avanti!» ritiene che, dato lo sfacelo economico in cui versa la Russia e il rischio che l'Intesa o la Germania fomentino nuovi tentativi controrivoluzionari, occorra raggiungere al più presto una stretta unione di tutti i partiti socialisti, superando le divergenze. Questa è considerata «la condizione sine qua non per la salvezza della rivoluzione» ${ }^{87}$.

Proprio per difendere il regime dei Soviet da attacchi interni ed esterni, il governo bolscevico decreta, agli inizi del 1918, l'istituzione di una milizia, fatto che solleva vivaci polemiche sulla stampa socialista, come dimostrano le accuse lanciate da Kautsky sulla nascita di un nuovo militarismo russo quale principale risultato della rivoluzione ${ }^{88}$. L'«Avanti!» difende la creazione di una forza armata, asserendo che l'esercito rosso è «lo strumento indispensabile» per affrontare un nuovo e «inevitabile» scontro con l'imperialismo tedesco: il trattato di pace consente di raccogliere le forze per la nuova lotta, che «non sarà solamente difensiva»" ${ }^{89}$ A differenza di Bordiga - che considera l'Armata rossa un mezzo esclusivamente di difesa e non di offesa ${ }^{90}$ Serrati non esclude che la Russia bolscevica stia meditando una rivincita militare contro l'ex nemico.

\footnotetext{
${ }^{84}$ A. G., «Utopia», in Avanti!, 25 luglio 1918, ora in GRAMSCI, Antonio, Il nostro Marx, cit., pp. 204-212.

${ }^{85}$ «Un anno di storia», in Il Grido del Popolo, 712, 1918; ora in GRAMSCI, Antonio, La Città futura, cit., pp. 734737.

${ }^{86}$ «Il terrore», in Critica Sociale, 19/1918, pp. 222-223.

${ }^{87}$ NADO, «Il pensiero del Congresso dei Soviets», in Avanti!, 25 marzo 1918; si veda anche: «Sulla Rivoluzione russa», in Avanti!, 30 giugno 1918.

${ }^{88}$ Cfr. SALVADORI, Massimo L., Kautsky fra ortodossia e revisionismo, in Storia del marxismo. II. Il marxismo nell'età della Seconda Internazionale, Torino, Einaudi, 1979, 4 voll., pp. 275-314.

${ }^{89}$ «La milizia territoriale», in Avanti!, 21 marzo 1918.

${ }^{90}$ Cfr. «La vittoria senz'ali», in Il Soviet, 16/1919.
} 
Su una cosa soltanto tutte le correnti del PSI concordano: nel condannare i tentativi da parte dell'Intesa di soffocare il regime dei Soviet, attraverso l'invio di truppe in varie zone strategiche del paese, col pretesto di difenderle da una eventuale offensiva tedesca ${ }^{91}$. Le proteste dei riformisti, tuttavia, derivano dal timore che il disegno reazionario dell'Occidente sospinga ancora di più i giovani verso le tesi leniniste, trasformando la rivoluzione in un mito, cosa che non potrebbe accadere se essa crollasse per le proprie deficienze interne ${ }^{92}$. Secondo l'«Avanti!», invece, il governo russo è il vero nemico di tutte le borghesie occidentali, comprese quelle delle Potenze centrali, in quanto rappresenta la possibilità materiale della sostituzione del proletariato alla borghesia nella gestione della società. Da qui, il rischio che i due belligeranti, pur continuando a fronteggiarsi sui campi di battaglia, collaborino attivamente per soffocare la rivoluzione ${ }^{93}$.

Poco prima che, nel settembre del 1918, inizi il congresso del Psi a Roma, arriva la notizia di un attentato a Lenin e, per alcuni giorni, si teme che il leader bolscevico sia morto: il massimo organo socialista ritiene che ciò non implicherebbe comunque la fine del regime dei Soviet, perché ormai «è radicato» nella coscienza del popolo ${ }^{94}$. Il congresso si apre inneggiando alla Russia e tra i compiti più urgenti del partito che vengono approvati, vi è quello di impedire il soffocamento della rivoluzione, paralizzando l'azione del governo con un inasprimento della lotta di classe ${ }^{95}$.

La minoranza riformista, invece, continua a ritenere l'ex Impero zarista un paese in preda al caos: sulla «Critica Sociale», Treves dichiara di non vedere alcuna ragione per cui «la tradizionale forma sindacalista-municipale della rivoluzione latina debba essere abiurata». L'essenza del marxismo consiste non nel porre un modello unico di rivoluzione, ma «nell'intendere nei fatti la formazione modale della rivoluzione, dovunque e sotto qualsiasi veste si manifesti» ${ }^{96}$.

\section{Conclusioni}

Con la fine della guerra, si verificano nuovi fermenti rivoluzionari che coinvolgono la Germania e l'Ungheria, episodi che rendono i bolscevichi fiduciosi di un'espansione rapida della rivoluzione in Europa ${ }^{97}$. Poiché i disordini coinvolgono i paesi sconfitti, i moderati italiani

\footnotetext{
${ }^{91}$ Cfr. CARETTI, Stefano, op. cit., pp. 152-153.

${ }^{92}$ Cfr. TREVES, Claudio, Dopo la vittoria, discorso del 27 novembre 1918; in ID., Come ho veduto la guerra, cit., pp. 171-189.

${ }_{93}$ Cfr. «Dubbi e riflessioni», in Avanti, 12 luglio 1918.

${ }^{94}$ «Perdura l'incertezza sulla sorte di Lenin», in Avanti, 3 settembre 1918. Lenin subì l'attentato ad opera della socialista rivoluzionaria Fanja Kaplan il 30 agosto 1918.

${ }^{95}$ Si veda: CORTESI, Luigi, Il socialismo italiano tra riforme e rivoluzione. Dibattiti congressuali del PSI. 1892-1921, Bari, Laterza, 1969, pp. 652-689.

${ }^{96}$ TREVES, Claudio, «Dopo il Congresso», in Critica Sociale, 18/1918, pp. 205-207.

${ }^{97}$ Cfr. PONS, Silvio, op. cit., pp. 22-23.
} 
guardano con scetticismo a tali fenomeni, considerandoli il prodotto della fame e della miseria, mentre i cambiamenti duraturi si attuano "per il crescere di coscienza e di volontà» tra le masse, che si muovono gradualmente verso la propria emancipazione ${ }^{98}$. Essi ritengono che le classi lavoratrici debbano sostenere il programma democratico di Wilson: il presidente americano può diventare «il pernio» dell'azione del proletariato, il quale deve battersi affinché i quattordici punti si applichino a tutti, vincitori e vinti ${ }^{99}$.

I massimalisti fanno invece propria la convinzione bolscevica che la guerra abbia accelerato il processo disgregativo del regime capitalistico, per cui in tutto l'Occidente i tempi sono maturi per lo sviluppo di nuovi ordinamenti ${ }^{100}$. A dividere, da questo momento, le due anime principali del partito, non è soltanto il tema dell'interpretazione dei fatti russi, ma quello della possibilità o meno della rivoluzione in Italia: la frattura, nei mesi successivi, diverrà sempre più acuta, fino alla rottura del 1921.

I riformisti si oppongono alla rivoluzione, perché il paese è povero di materie prime e dipende dall'estero per la maggior parte dei suoi rifornimenti, quindi è probabile che l'Intesa ricorrerà al blocco economico per spegnere l'insurrezione, in seguito alla quale i socialisti saranno messi fuori della legge $\mathrm{e}^{101}$. Essi sostengono l'ipotesi della Costituente, ma la Direzione del PsI la scarta all'unanimità e lancia alle masse le parole d'ordine della dittatura del proletariato e della repubblica socialista ${ }^{102}$.

Tuttavia, manca una strategia di lotta che dia concretezza alle istanze rivoluzionarie: nei due anni successivi alla fine della guerra, mentre si moltiplicano gli scioperi e le agitazioni popolari, il gruppo dirigente del PSI resterà prigioniero di un massimalismo puramente verbale, senza riuscire a tradurre in indicazioni pratiche gli insegnamenti di Lenin. Anche Bordiga, pur rilevando acutamente le deficienze della compagine socialista, il suo essere sostanzialmente una grande macchina elettorale, non saprà andare oltre la proposta di un rigido astensionismo quale strumento atto a preparare le masse a insorgere ${ }^{103}$.

Gramsci, invece, comprende che il fatto essenziale della rivoluzione russa è l'instaurazione di un nuovo tipo di Stato, «lo Stato dei Consigli» ${ }^{104}$, di natura proletaria, e insieme a un gruppo di operai e intellettuali raccolto intorno alla rivista L'«Ordine Nuovo», nel tentativo di ritradurre la lezione bolscevica nella situazione italiana, dà vita a Torino al movimento dei Consigli di fabbrica.

\footnotetext{
${ }^{98}$ ID., «La pace rivoluzionaria», in Critica Sociale, 22/1918, pp. 253-255.

${ }^{99}$ ID., «Wilson», in Critica Sociale, 20/1918, pp. 229-230.

${ }^{100}$ Si veda: SABBATUCCI, Giovanni, I socialisti nella crisi dello Stato liberale (1918-1926), in ID., Storia del socialismo italiano, Vol. III, Guerra e dopoguerra 1914-1926, Roma, Il Poligono, 1982, pp. 143-161.

${ }^{101}$ Ibidem.

${ }^{102}$ Cfr. AMBROSOLI, Luigi, Né aderire, né sabotare, Milano, Ed. Avanti!, 1915-1918, pp. 315-316.

${ }^{103}$ Cfr. SAVANT, Giovanna, Bordiga, Gramsci e la Grande Guerra (1914-1920), Napoli, La Città del Sole, 2016, pp. 273-281.

104 «Leninismo e marxismo di Rodolfo Mondolfo», in L'Ordine Nuovo, 2/1919.
} 
I nuovi istituti proletari si basano sull'autogoverno degli operai-produttori ${ }^{105}$ e pur non costituendo una rigida traduzione italiana dei Soviet, rappresentano probabilmente, con tutti i loro limiti, l'unico tentativo concreto di applicare la lezione di Lenin nel nostro paese, prima dell'avvento del fascismo.

${ }^{105}$ Si veda: DE FELICE, Franco, Serrati, Bordiga, Gramsci e il problema della rivoluzione in Italia, Bari, De Donato, 1971, pp. 275-311. 


\section{L'AUTORE}

Giovanna SAVANT è Dottoressa di ricerca in Studi politici e membro del Comitato Scientifico dell'Istituto Gramsci di Torino, col quale collabora attivamente. Ha scritto alcuni saggi su Antonio Gramsci e sull'elitismo apparsi in volumi collettanei e su riviste. Ha pubblicato i seguenti libri: Intransigenti e collaborazionisti. Serrati e Treves davanti alla Grande Guerra, Roma, Aracne, 2013; Bordiga, Gramsci e la Grande Guerra (1914-1920), Napoli, La Città del Sole, 2016.

URL: < http://www.studistorici.com/progett/autori/\#Savant > 